\title{
ORIGINAL
}

\section{ANALISIS COSTE-EFECTIVIDAD DE DISTINTOS MÉTODOS DE DIAGNÓSTICO POR IMAGEN DEL TROMBOEMBOLISMO PULMONAR AGUDO}

\author{
Stella Maris Batallés (1), Roberto Lisandro Villavicencio (1) y Stella Maris Pezzotto (2) \\ (1) Instituto Cardiovascular de Rosario - Fundación “Dr. J. R. Villavicencio". Rosario. Argentina. \\ (2) Consejo de Investigaciones. Universidad Nacional de Rosario. Argentina.
}

\section{RESUMEN}

Fundamento. La técnica diagnóstica óptima para detectar tromboembolismo pulmonar agudo (TEP) continúa en discusión. La gammagrafía pulmonar de ventilación/perfusión ha sido el examen preferido durante décadas, pero con el advenimiento de nuevas pruebas de imágenes las posibilidades diagnósticas se ampliaron, siendo necesario evaluarlas desde la perspectiva del coste y de la efectividad. El objetivo de este trabajo fue evaluar distintos métodos de diagnóstico por imagen para detectar TEP agudo para determinar el más coste-efectivo.

Métodos. Análisis de coste-efectividad (CE) empleando un árbol de decisiones para modelar distintas pruebas (centellograma V/Q, TC helicoidal, angiografía por tomografía computada multidetector (TCMD), resonancia magnética por imágenes (RMI) y arteriografía convencional). Se obtuvieron valores de sensibilidad, especificidad, valor predictivo positivo (VPP) y negativo (VPN) de las pruebas diagnósticas. Resultado medido: "caso detectado de TEP". Los costes evaluados fueron los directos, expresados en euros $(€)$, incluyendo los secundarios a las complicaciones de los métodos diagnósticos. Se realizó un análisis de sensibilidad de una vía para evaluar la robustez de las conclusiones.

Resultados. No se eliminaron pruebas por dominancia extendida. La tasa cruda de CE para TCMD fue de $486 €$ por cada caso de TEP detectado. El coste marginal entre la TC helicoidal y el centellograma V/Q fue de $103 €$ para detectar 8 casos adicionales de TEP, mientras que el coste marginal entre la TCMD y la TC helicoidal fue de $229 €$ para detectar un caso adicional de TEP.

Conclusiones. La prueba diagnóstica más coste-efectiva fue la TCMD, hallazgo que mostró robustez en el análisis de sensibilidad. Sin embargo, el análisis de C-E incremental nos mostró que la TCMD costó $229 €$ más respeto a la TC helicoidal para lograr una mínima mejora en la efectividad de la prueba (detección de TEP agudo). El alto valor predictivo negativo de la TCMD justificaría su realización en casos de sospecha de TEP de ramas periféricas de pequeño calibre.

Palabras clave: Coste efectividad. Tromboembolismo pulmonar. Diagnóstico.

Correspondencia:

Stella M. Batallés

Oroño 450 - 2000

Rosario. Argentina

batalless@icronline.com

\section{ABSTRACT \\ Cost-Effectiveness Analysis of Different Imaging for the Diagnosis of Acute Pulmonary Embolism}

Background. The optimal diagnostic test for detecting acute pulmonary embolism (APE) is still under discussion. The ventilation/perfusion scanning has been the preferred examination for several decades, but with the development of new tests the diagnostic posibilities have increased. It is necessary to evaluate them from the cost-effectiveness perspective. The goal of this study was to evaluate several methods of imaging diagnosis so as to determine the most cost-effective for detecting APE.

Methods. Cost-effectiveness (CE) analysis using a decision tree to model various diagnostic test (V/Q lung scan, spiral CT, angiography by MDCT, MRI and conventional arteriography). Sensitivity and specificity values, and positive and negative predictive values of diagnostic tests were calculated. Expected outcome: "new APE case detected." The direct costs were evaluated in Euros $(€)$, including the secondary complications of diagnostic methods. To assess the robustness of the findings, a one way sensitivity analysis was performed.

Results. The most cost-effective diagnostic test was angiography by MDCT. No diagnostic test were eliminated by extended dominance. The crude rate of CE for MDCT was $486 €$ per case of APE detected. The marginal cost between spiral CT and V / Q lung scan was $€ 103$ for 8 cases of APE detected additionally, while the marginal cost between MDCT and spiral CT was $229 €$ to detect an additional case of APE.

Conclusions. The most cost-effective diagnostic test was the MDCT; this finding showed to be robust in relationship to sensitivity, specificity and costs changes. However, the incremental C-E analysis showed that MDCT was capable to detect only one additional case of APE than spiral CT, with an incremental cost of $229 €$. When peripheral branches are affected, high negative predictive value of the MDCT justify its conduct.

Key words: Cost effectiveness. Thromboembolisms, pulmonary. Diagnosis.

No existen conflictos de intereses. 


\section{INTRODUCCION}

El tromboembolismo pulmonar (TEP) consiste en la oclusión total o parcial de la circulación pulmonar, ocasionada por un coágulo sanguíneo proveniente de la circulación venosa sistémica, incluidas las cavidades cardíacas derechas y que, dependiendo de su magnitud, puede o no originar síntomas $^{1,2}$. Constituye la tercera causa de enfermedad cardiovascular aguda en orden de frecuencia, después del infarto de miocardio y del accidente cerebrovascular, siendo responsable de 200.000 a 300.000 hospitalizaciones en los Estados Unidos ${ }^{3,4}$. La mortalidad del TEP agudo sin tratamiento es del $30 \%{ }^{5-7}$. Por lo tanto, es necesario detectar la enfermedad de manera rápida y precisa para enfocar el manejo terapéutico del paciente ${ }^{4}$.

La técnica diagnóstica óptima para detectar TEP agudo en la práctica clínica de la mayoría de los hospitales continúa en discusión ${ }^{8}$.

En nuestros días, la tendencia mundial es lograr la mayor objetividad en la toma de decisiones sobre los recursos disponibles en el sector salud. Los estudios de coste efectividad (CE) constituyen una herramienta para disminuir la incertidumbre en la toma de decisiones en el ámbito sanitario.

El análisis de CE es un tipo de evaluación económica en el que se comparan dos o más intervenciones sanitarias en términos de unidades naturales de efectividad. Se trata de contrastar estrategias que comparten los mismos objetivos (en nuestro caso diagnóstico de TEP agudo), cuando sus niveles de efectividad difieren. Combina la información de la efectividad clínica de las estrategias con la de los costes de las mismas. La medida central que resume dicha relación es la tasa de CE.

El caso del TEP agudo es paradigmático, ya que existen numerosos métodos de diagnóstico por imágenes que intentan detectarlo. Algunos de ellos son muy buenos para descartarlo, otros los son para confirmarlo y otros podrían lograr ambas cosas, pero frecuentemente son "sub-diagnósticos"9.

La gammagrafía pulmonar de ventilación/ perfusión ha sido considerada como el estudio de primera línea para investigar TEP agudo. Un estudio de "alta probabilidad", especialmente en un contexto clínico sugestivo, ha mostrado alta asociación con angiografía pulmonar positiva. Un estudio "normal" prácticamente excluye el diagnóstico de TEP agudo ${ }^{6}$. Pero en todas las situaciones intermedias, el centellograma V/Q tiene dificultades para detectar o excluir esta entidad.

La tomografía computada helicoidal $(\mathrm{TCH})$, por su parte, es una modalidad diagnóstica disponible en muchas instituciones en nuestros días y es otra de las opciones útiles para evaluar pacientes con sospecha de TEP agudo. Sin embargo, este método, que emplea radiaciones ionizantes y contraste yodado endovenoso, tiene insuficiente sensibilidad para detectar trombos en las ramas pulmonares periféricas ${ }^{8}$.

La tomografía computada multidetector (TCMD) ha demostrado una alta sensibilidad y especificidad para detectar trombos en todos los vasos pulmonares, incluidos los de muy pequeño calibre (subsegmentarios $)^{4,10}$. Los cortos tiempos de adquisición y la consiguiente reducción de artefactos por movimientos cardíacos son ventajas importantes respecto a la $\mathrm{TCH}$.

El empleo de resonancia magnética por imágenes (RMI) para la detección de trombos pulmonares resulta atractivo ya que es un método diagnóstico que permite la evaluación de la vasculatura pulmonar sin necesidad de inyectar sustancia de contraste yodada endovenosa, con el menor riesgo de reacción anafiláctica para el paciente y que evita la exposición a la radiación ${ }^{9}$.

La arteriografía pulmonar es conocida como el "patrón de oro" para el diagnóstico 
de TEP agudo. Sin embargo, lo invasivo del método, la posibilidad de producir reacciones anafilácticas y complicaciones cardiovasculares ${ }^{4}$, sumado a la baja concordancia interobservador en la interpretación de trombos en arterias subsegmentarias aún con arteriografía selectiva, hace dudar de su condición de técnica de referencia ${ }^{11,12}$.

El objetivo del presente trabajo fue valuar qué estrategia resulta la más costeefectiva para detectar TEP agudo.

\section{MATERIAL Y METODOS}

Se realizó un análisis de coste-efectividad (CE) desde la perspectiva del tercer pagador.

Se consideró el escenario de aquel paciente que estando hospitalizado por otro diagnóstico distinto a TEP presentó un epi- sodio de disnea súbita que requirió descartar dicha entidad.

Fueron evaluados los costes de distintas técnicas de imagen para diagnóstico de TEP agudo y se compararon con el resultado deseado: "caso detectado de TEP agudo".

Las técnicas en evaluación fueron: centellograma V/Q, TCH, angiografía por TCMD (considerándose por igual todas las generaciones de 4 a 256 filas de detectores), RMI pulmonar y arteriografía pulmonar convencional.

Se revisó la literatura médica para obtener valores de sensibilidad, especificidad, valor predictivo positivo y negativo de las técnicas en consideración. Cabe aclarar que dichos valores no se obtuvieron de metaanálisis ni de revisiones sistemáticas sino de diversas fuentes bibliográficas (tabla 1).

Tabla 1

Supuestos clínicos

\begin{tabular}{|l|c|c|c|}
\hline Variable & Valor & Rango & Referencias \\
\hline Prevalencia de TEP agudo & 28,4 & $15-50$ & 23 \\
\hline Probabilidad de TEP agudo en cetellograma V/Q, con TEP presente & & & 23 \\
\hline Normal & 2,0 & & \\
\hline Baja & 15,9 & & \\
\hline Intermedia & 41,2 & & \\
\hline Alta & 40,9 & & \\
\hline Probabilidad de TEP agudo en centellograma V/Q, con TEP ausente & 23 & & \\
\hline Normal & 19,4 & & \\
\hline Baja & 40,3 & & \\
\hline Intermedia & 38,0 & & $24-16$ \\
\hline Alta & 2,3 & & $24-16$ \\
\hline Sensibilidad TCH & 88,6 & $63-95$ & 25 \\
\hline Especificidad TCH & 92,5 & $78-97$ & \\
\hline Sensibilidad TCMD & 96,0 & $90-100$ & \\
\hline Especificidad TCMD & 97,0 & $90-100$ & \\
\hline Sensibilidad RMI pulmonar & 38,5 & $39-49$ & 27 \\
\hline Especificidad RMI pulmonar & 82,4 & $50-90$ & \\
\hline Sensibilidad arteriografía pulmonar & 98,0 & $86-100$ & \\
\hline Especificidad arteriografía pulmonar & 97,0 & $95-98$ & 28 \\
\hline
\end{tabular}

Abreviaturas

TEP: tromboembolismo pulmonar

Centellograma V/Q: centellograma ventilación-perfusión

TCH: tomografía computada helicoidal

TCMD: tomografía computada multidetector

RMI: resonancia magnética por imágenes 
Con los supuestos estadísticos obtenidos (tabla 1) se generó un árbol de decisión para comparar las técnicas en evaluación, empleando un software específico (Data Treeage 3.5). De cada técnica evaluada se obtuvo el coste neto y la efectividad neta. Las técnicas fueron independientes unas de otras.

Para efectuar el análisis de CE en primer lugar se ordenaron las técnicas en orden creciente de coste. Se analizó la CE incremental entre las técnicas incluidas con el fin de evidenciar dominancia fuerte (técnica con menor coste y mayor efectividad o con menor efectividad y menor coste), dominancia débil (técnicas con mayor efectividad pero igual coste, igual efectividad y mayor coste, menor efectividad e igual coste o menor coste e igual efectividad) o dominancia extendida (técnicas con mayor coste y mayor efectividad, pero menor tasa de C-E incremental) de alguna de ellas. Finalmente, se analizó la CE incremental de las técnicas que quedaron en el modelo.

Los costes analizados fueron sólo los directos. También se incluyeron los secundarios a las posibles complicaciones de las pruebas que emplearon contraste yodado endovenoso (TCH, TCMD y Arteriografía pulmonar); se consideraron los costos de alergia al mismo y de la diálisis por falla renal post-inyección (si bien hoy en día es infrecuente esta complicación) (tabla 2).
Los costes se expresaron en euros () y la fuente de información fueron los aranceles sugeridos por la Cámara de Entidades de Diagnóstico y Tratamiento Ambulatorio de Argentina por una parte y por fuentes locales (pertenecientes a centros de costes de prestadores privados), con aranceles actualizados a Junio de 2008. El coste de la diálisis por fallo renal proviene del valor promedio que los financiadores pagan por dicha entidad nosológica (valor modulado que considera prestaciones y honorarios profesionales y derechos institucionales). No se midieron costes indirectos como pérdidas de días de trabajo por hospitalización del paciente o gastos o pérdidas económicas de la familia por atención del paciente) y no se aplicó tasa de descuento a los costes ni a los efectos.

Para evaluar la robustez de las conclusiones del estudio se realizó un análisis de sensibilidad de una vía de los valores de sensibilidad y especificidad de los métodos de imagen, como también de los costes de las distintas técnicas.

\section{RESULTADOS}

Con la RMI fueron necesarios $585 €$ para detectar un nuevo caso de TEP, mientras que con la arteriografía pulmonar convencional fueron necesarios $679 €$ para

Tabla 2

Coste en euros de las diferentes técnicas diagnósticas (incluyendo las posibles complicaciones de las mismas)

\begin{tabular}{|l|c|c|c|c|c|}
\hline Costo & Centellograma V/Q & TCH & TCMD & RMI & Arteriografía Pulmonar \\
\hline Estudio* & 154 & 187 & 416 & 489 & 553 \\
\hline Alergia & & 4,4 & 4,4 & 4,4 & 4,4 \\
\hline Dialisis & & 66 & 66 & & 66 \\
\hline Cateteres & 154 & & & & 656,4 \\
\hline $\begin{array}{l}\text { Costo Total } \\
\text { Estrategia }\end{array}$ & & 257,4 & 486,4 & 493,4 & \\
\hline
\end{tabular}

* Incluye costes de contraste yodado endovenoso (en los casos de TCH, TCMD y Arteriografía pulmonar) y de gadolinio endovenoso (en el caso de RMI) Abreviaturas

Centellograma V/Q: centellograma ventilación-perfusión

TCH: tomografía computada helicoidal

TCMD: tomografía computada multidetector

RMI: resonancia magnética por imágenes 
Tabla 3

Análisis de coste-efectividad marginal

\begin{tabular}{|l|c|c|c|c|c|c|}
\hline Prueba & Coste en euros & Cost Marg en euros & Efect & Efect Marg & C/E* & C/E Marg* \\
\hline CENT, V/Q & 154 & & 0,1 & & 2.758 & \\
\hline TCH & 257,4 & 103,4 & 0,9 & 0,8 & 296 & 127,2 \\
\hline TCMD & 486,4 & 229 & 1,0 & 0,1 & 499 & $2.168,4$ \\
\hline RMI & 493,4 & 7 & 0,8 & $-0,1$ & 585 & (Dominada) \\
\hline ARTER & 656,4 & 170 & 1,0 & $-0,0$ & 679 & (Dominada) \\
\hline
\end{tabular}

* C/E y C/E Marg: euros por caso detectado de TEP agudo

Abreviaturas

Cost Marg: coste marginal

Efect: efecto

Efect Marg: efecto marginal

C/E: costel efectividad

C/E Marg: costel efectividad marginal

Cent. V/Q: centellograma ventilación-perfusión

TCH: tomografía computada helicoidal

TCMD: tomografía computada multidetector

RMI: resonancia magnética por imágenes

Arter: arteriografía pulmonar

detectarlo. Ambas resultaron las pruebas de mayor coste y menor efectividad, por lo que fueron excluidas del modelo.

Después de excluir las pruebas dominadas quedaron en el modelo el centellograma V/Q, TCH y la angiografía por TCMD.

No se eliminaron pruebas que demostrasen mayor coste y mayor efectividad, pero menor tasa de CE incremental (situación conocida como dominancia extendida).

La angiografía por TCMD fue la prueba más costo-efectiva. La tasa cruda de $\mathrm{CE}$ para TCMD fue de $486 €$ por cada caso de TEP detectado.

El coste marginal entre la TCH y el centellograma V/Q fue de $103 €$ para detectar 8 casos adicionales de TEP, mientras que el coste marginal entre la TCMD y la TCH fue de $229 €$ para detectar un caso adicional de TEP (tabla 3).

No se evidenciaron cambios en los resultados obtenidos al variar los valores de sensibilidad y especificidad de los métodos de imagen (valores de los rangos expuestos en la tabla 1) y de los costes de las distintas pruebas $( \pm 100 €$ de los establecidos en la tabla 2$)$.
Figura 1
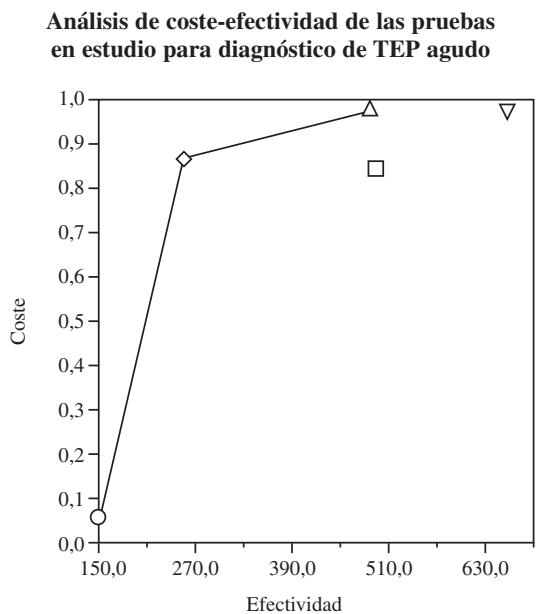

$$
\begin{aligned}
& \text { O Centellograma V/Q } \\
& \diamond \mathrm{CTH} \\
& \triangle \text { Angio TCMD } \\
& \square \text { RMI } \\
& \nabla \text { Arteriografía }
\end{aligned}
$$

\section{DISCUSION}

En nuestro modelo, con los supuestos antes definidos, la angiografía por TCMD fue la prueba más coste-efectiva. Esto significa que, a igual efectividad que la arterio- 
grafía pulmonar, su coste fue menor al de esta última.

Antes de la aparición de la TCH, el algoritmo de estudio que aparecía como más coste-efectivo para diagnóstico de TEP agudo era centellograma pulmonar V/Q más evaluación ecográfica de venas inferiores y angiografía pulmonar ${ }^{6,13}$. En nuestro análisis, exceptuando el centellograma pulmonar V/Q que mostró la menor efectividad, el resto de las modalidades diagnósticas mostraron un alto valor de efectividad, siendo el coste el factor clave en la relación entre las variables coste y efectividad.

En la investigación de van Erkel y col. ${ }^{14}$ se estimó "sobrevida" como unidad de efectividad y costes de 12 pruebas que combinaban $\mathrm{TCH}$, centellograma $\mathrm{V} / \mathrm{Q}$, ecografía vascular venosa, angiografía convencional y/o determinación bioquímica de dímero $\mathrm{D}$. Los autores concluyeron que la prueba más coste-efectiva para diagnóstico de TEP era la determinación de dímero D seguida por $\mathrm{TCH}$ si aquel era positivo. En nuestra experiencia, al no ser solicitado de forma rutinaria en todos los pacientes, no se consideró el coste del dímero D en cada una de las pruebas.

Es conocida la limitación de la $\mathrm{TCH}$ para detectar trombos en las ramas subsegmentarias pulmonares ${ }^{15,16}$. Sin embargo, existen estudios que sugieren que la detección de los trombos en las ramas pulmonares periféricas es de escasa utilidad clínica y su falta de detección puede ser tolerada ${ }^{17}, 18$. Pero, con el advenimiento de la TCMD en los últimos años, se ha demostrado que su valor predictivo negativo es muy alto, con valores de hasta un $99 \%{ }^{19}$.

A diferencia de van Erkel y col. y de varias experiencias publicadas ${ }^{20,21}$, en nuestro estudio no utilizamos una medida de efectividad final -como por ejemplo son "años de vida ganados" o "sobrevida" 22 . Sin embargo, creemos que el resultado intermedio "casos detectados de TEP agudo" ("output" intermedio) es relevante y está acorde al objetivo de la investigación que fue determinar la prueba de mayor $\mathrm{CE}$ para detectar dicha entidad patológica.

Creímos erróneo comparar la razón de C-E de una prueba diagnóstica con otra y optar por aquella intervención cuya razón resultase más baja, ya que analizamos pruebas excluyentes entre sí. En nuestra experiencia, fue necesario efectuar el análisis de CE incremental; es decir, cuánto se incrementó el efecto (nuevos casos de TEP agudo detectados) y cuánto se incrementaron los costes en comparación con la opción inmediatamente anterior en orden de coste. Dicho de otra forma, el análisis de C-E incremental nos permitió saber cuánto costó cada caso detectado de TEP agudo por encima de lo que se logró diagnosticar con la prueba inmediatamente más económica. De dicho análisis de CE incremental observamos que la tasa cruda de C-E para TCMD fue de $486 €$ por cada caso de TEP detectado y que el coste marginal entre la TCH y el centellograma V/Q fue de $103 €$ para detectar 8 casos adicionales de TEP, mientras que el coste marginal entre la TCMD y la TCH fue de $229 €$ para lograr detectar sólo un caso adicional de TEP.

No consideramos necesario aplicar tasa de descuento a los valores de coste y efectividad dese el momento que no obtuvimos medidas de efectividad finales, sino intermedias, con un horizonte temporal inmediato.

Al plantear el escenario de aquel paciente que estando hospitalizado por otro diagnóstico distinto a TEP presenta un episodio de disnea súbita que requiere descartarlo, no se consideraron los costes de la hospitalización.

Limitaciones del estudio: Al utilizar un modelo probabilístico como base de análisis, la validez interna de nuestro estudio fue 
menor que si se tratara de una evaluación económica concurrente a un ensayo clínico o basada en una revisión sistemática de ensayos clínicos (aunque éstos últimos son más rigurosos desde el punto de vista metodológico y tienen mayor validez interna, presentan menor validez externa).

Nuestro trabajo implicó la aplicación de un modelo matemático que no contempló cuadros clínicos o pacientes particulares. En muchas oportunidades el contexto clínico, el medio socio-económico, la ubicación geográfica, la disponibilidad de la última tecnología entre otros factores, son decisivos a la hora de seleccionar un método de diagnóstico por imágenes para evaluar TEP agudo.

Si bien es real que la mayoría de los centros sanitarios de Europa y Estados Unidos ya cuenta con equipos de TCMD, que han reemplazado a la $\mathrm{TCH}$, donde lo que varía es la cantidad de filas de detectores, la situación no es la misma en América Latina, donde ambas tecnologías conviven. Sería quizás más oportuno incluir en el análisis de CE la angiografía por TCMD con 4 filas de detectores, con 16, con 64, con 128 y con 256 separadamente.

Respecto a los resultados obtenidos, no es posible realizar extrapolaciones de los mismos fuera del ámbito local del estudio debido a los valores de los costes y de la efectividad incluidos en el análisis.

Por último, cabe la duda de conocer qué ocurriría con la C-E de las pruebas valoradas en distintos subgrupos de pacientes, donde las efectividades pueden modificarse de acuerdo a determinadas variables (por ejemplo, edad de los pacientes, sexo, factores de riesgo asociados, etc.).

Como conclusión, la prueba diagnóstica más coste-efectiva en nuestra experiencia fue la TCMD, hallazgo que mostró robustez ante las variaciones de sensibilidad, especificidad y coste de las pruebas incluidas en el análisis.
Sin embargo, el análisis de CE incremental nos mostró que, respecto a la TCH, la TCMD costó $229 €$ más para lograr una mínima mejora en la efectividad de la prueba (detección de TEP agudo). El alto valor predictivo negativo de la TCMD justificaría su realización en casos de sospecha de TEP de ramas periféricas de pequeño calibre.

No se observaron pruebas cuyas efectividades fuesen menores a cero.

Tanto la RMI pulmonar como la arteriografía pulmonar convencional mostraron mayor coste y menor e igual efectividad respectivamente que la angiografía por TCMD, siendo ambas dominadas por la angiografía por TCMD (tabla 3) (figura 1).

Con vistas a los nuevos escenarios mundiales, donde la disponibilidad de tomógrafos computados de distintas filas de detectores es cada vez mayor, sería más conveniente incluir en el análisis de CE la angiografía por TCMD con 4 filas de detectores, con 16, con 64, con 128 y con 256 separadamente.

\section{BIBLIOGRAFIA}

1. Amoliga A, Matthay M, Matthay R. Pulmonary Thromboembolism and other pulmonary vascular diseases. En: George RS, Light RW, Matthay MA, et al. Chest Medicine: Essentials of Pulmonary and Critical Care Medicine. Baltimore: Williams \& Wilkins; 1995. pp. 130-135.

2. Fitzmaurice DA, Hobbi R. Tromboembolism. En: A compendium of the Best evidence for effective health care. London: Clinical Evidence, ACP/ASIM-BMJ Publishing Group eds.; 1999. pp. 130-135.

3. Wittram C. How I do it: CT pulmonary angiography. AJR. 2007; 188: 1255-1261.

4. Kazerooni E, Gross B. Radiología cardiopulmonar. $1^{\text {a }}$. ed. Buenos Aires: Ediciones Journal; 2006. pp. 581-597.

5. Dalen JE. When can treatment be withheld in patients with suspected pulmonary embolismo?. Arch Intern Med. 1993; 153: 1415-1418. 
6. Paterson DI, Schwartzman K. Diagnostic test Incorporating Spiral CT for the Diagnosis of Acute Pulmonary Embolism: A Costeffectiveness Analysis. Chest. 2001; 119: 17911800 .

6. Pineda LA, Hathwar VS, Grant BJ. Clinical suspicion of fatal pulmonary embolism. Chest. 2001; 120: $791-5$

7. Quiroz R, Kucher N, Zou KH y col. Clinical Validity of a Negative Computed Tomography Scan in Patients With Suspected Pulmonary Embolism: A Systematic Review. JAMA. 2005; 293(16): 2012 2017.

8. Kearon C. Diagnosis of pulmonary embolism. CMAJ. 2003; 168 (2): 183-194.

9. Eyer BA, Goodman LR, Washington L. Clinicians' Response to Radiologists' Reports of Isolated Subsegmental Pulmonary Embolism or Inconclusive Interpretation of Pulmonary Embolism Using MDCT. AJR. 2005; 184: 623-628.

10. Stein PD, Henry JW, Gottschalk A. Reassessment of pulmonary angiography for the dignosis of pulmonary embolism: relation of interpreter agreement to the order of the involved pulmonary arterial branch. Radiology. 1999; 210: 689-91.

11. Uresandia F, Blanquerb J, Congetc F, de Gregorioc MA, Lobod JL, Oteroe $\mathrm{R}$ et al. Guía para el diagnóstico, tratamiento y seguimiento de la tromboembolia pulmonar. Arch Bronconeumol. 2004; 40 (12): 580-94.

12. Oudkerk M, van Beek EJR, van Putten WLJ et al. Cost-effectiveness analysis of various diagnostic test in the diagnostic management of pulmonary embolism. Arch Intern Med. 1993; 153: 947-954.

13. van Erkel AR, van Rossum AB, Bloem JL et al. Spiral CT angiography for suspected pulmonary embolismo: a cost-effectiveness analysis. Radiology. 1996; 201: 29-36.

14. van Rossum AB, Pattynama PMT, Ton ERTA et al. Pulmonary embolism: validation of sipral CT angiography in 149 patients. Radiology. 1996; 201: 467-470.

15. Mayo JR, Remy-Jardin M, Muller NL et al. Pulmonary embolism: prospective comparison of spiral CT with ventilation-perfusion scintigraphy. Radiology. 1997; 205: 447-452.

16. Gurney GW. No fooling around: direct visualization of pulmonary embolism (editorial). Radiology. 1993; 188: 618-619.
17. Remy-Jardin M, Remy J, Deschildre F et al. Diagnosis of pulmonary embolism with sipral CT: comparison with pulmonary angiography and scintigraphy. Radiology. 1996; 200: 699-706.

18. Kavanagh EC, O'Hare A, Hargaden G et al. Risk of pulmonary embolism after negative MDCT pulmonary angiography findings. AJR 2004; 182:499-504.

19. Hull RD, Feldstein W, Stein PD et al. Cost-effectiveness of pulmonary embolism diagnosis. Arch Intern Med. 1996; 156: 68-72.

20. Doyle N, Ramirez M, Mastrobattista JM, Monga M, Wagner L, Gardner M. Diagnosis of pulmonary embolism: a cos-effectiveness analysis. American Journal of Obstetrics and Gynecology. 2004; 191: 1019-1023.

21. Drummond M, O’Brien B, Stoddart G, Torrance G. Methods for the Economic Evaluation of Health Care Programmes. $2^{\text {nd }}$ ed. New York: Oxford Medical Publications; 1997. pp. 96-138.

22. The PIOPED Investigators. Value of ventilation/perfusion scan in acute pulmonary embolism: results of the Prospective Investigation of Pulmonary Embolism Diagnosis (PIOPED). JAMA. 1990; 263: 2753-2759.

23. Goodman LR, Curtin JJ, Mewissen MW, et al. Detection of pulmonary embolism in patients with unresolved clinical and scintigraphic diagnosis: helical CT versus angiography. AJR Am J Roentgenol. 1995; 164: 1369-1374.

24. Stein PD, Athanasoulis C, Alavi A, et al. Complications and validity of pulmonary angiography in acute pulmonary embolism. Circulation. 1992; 85: 462-468.

25. Turkstra F, Kuijer PMM, van Beek EJR, et al. Diagnostic utility of ultrasonography of leg veins in patients suspected of having pulmonary embolism. Ann Intern Med. 1997; 126: 775-781.

26. Wells PS, Ginsberg JS, Anderson DR, et al. Use of a clinical model for safe management of patients with suspected pulmonary embolism. Ann Intern Med. 1998; 129: 997-1005.

27. Cogo A, Lensing AWA, Wells P, et al. Noninvasive objective tests for the diagnosis of clinically suspected pulmonary embolism. Haemostasis. 1995; 25: 27-39.

28. Bautista Bautista E, Gutiérrez Fajardo P, Ramírez A, Hernández Hernández J. Diagnóstico de la tromboembolia pulmonar. Gac Méd Méx. 2007; 143 (1) : 19-24. 